\title{
Investigation of sour substances by a set of all-solid-state membrane electrodes
}

\author{
M. Szpakowska* ${ }^{\mathrm{a} *}$ E. Marjańska ${ }^{\mathrm{a}}$, A. Lisowska-Oleksiak ${ }^{\mathrm{b}}$ \\ ${ }^{a}$ Commodity Science Laboratory, Faculty of Economics and Management, Gdansk University of Technology, \\ Narutowicza str. 11/12, 80-952 Gdansk, Poland \\ email:mszpak@pg.gda.pl; ewam@zie.pg.gda.pl \\ ${ }^{\mathrm{b}}$ Department of Chemical Technology, Chemical Faculty, Gdansk University of Technology, \\ Narutowicza str.11/12, 80-952 Gdansk, Poland \\ email: alo@chem.pg.gda.pl
}

Received 27 August 2007; revised 19 December 2007; accepted 26 December 2007

\begin{abstract}
All-solid-state electrodes were tested in contact with sour taste electrolytes. The tested electrodes consist of two types of membranes. The first sub-layer covering directly the glassy carbon disc is an electroactive polymer, poly(3,4-ethylenedioxythiophene), used as ion-to-electron transducer. The influence of thickness of this membrane on electrode response was examined. The second, outer layer of the sensor, is an ion selective lipophilic compound-polymer membrane. The influence of the thickness and mass ratio of lipophilic compound/polyvinyl chloride of this second layer on the sensor response was also examined. It was found that the thickness of the electroactive polymer has no effect on sensitivity and stability of electrodes used. On the contrary, increase of the thickness of lipophilic compound-polymer membrane causes decrease of sensitivity of electrodes to sour substances and improvement of their stability. It was found that all-solid-state electrodes with positively charged and neutral polyvinyl chloride membranes behave similarly in full contradiction to traditional ion selective electrodes with inner solution. The response of all-solid-state electrodes with positively charged membranes to hydrochloric acid is linear independently on mass ratio of lipohilic compound/polyvinyl chloride used. This suggests that they might work for $\mathrm{pH}$ determination.
\end{abstract}

Keywords: All-solid-state electrodes; Taste sensor; Polymer membranes; Electroactive polymer

*Corresponding author.

Presented at the Third Membrane Science and Technology Conference of Visegrad Countries (PERMEA), Siofok, Hungary, 2-6 September 2007. 


\section{Introduction}

Taste is composed of five basic taste qualities: sourness, bitterness, saltiness, sweetness and umami [1]. The first one is produced by hydrogen ions (e.g. citric, acetic and hydrochloric acids). Bitterness is caused usually by quinine hydrochloride or caffeine, whereas saltiness is due to sodium chloride. Sweetness is caused by different sugars (e.g. sucrose, glucose, fructose) or by so called "artificial sweeteners" (e.g. aspartame). The taste umami is used to designate a pleasant gustatory sensation that is qualitatively different from sour, bitter, salty and sweet. This taste is produced mainly by monosodium glutamate or disodium guanylate [1].

The taste of foodstuff is usually estimated by organoleptic methods which are based on human senses [2]. When taste substance is reaching the biological membrane of gustatory cells in taste buds on the tongue the information is transformed into an electric signal. It is transmitted along a nerve fiber to a brain, where it is perceived [1]. The taste estimated by organoleptic methods is influenced by many factors from which the nature of living organism and its sensitivity threshold play an important role. This means that organoleptic methods cannot be objective enough for quality control of foodstuffs. Therefore, an elaboration of instrumental taste sensors for estimation and/or quality control of products is strongly desired in food industry.

Recently, many investigations are focused on elaboration of a taste sensor [3-10]. One of them is potentiometric taste sensor composed of seven or eight lipid-polymer membrane electrodes transforming information of taste substances into electric signals [3]. This sensor was used for recognition of similar products from different brands and for evaluation of taste of foodstuffs such as beer, sake, coffee, mineral water, tomatoes, etc. [3]. Its commercialized versions, i.e. Taste Sensing Systems SA401A and SA402B are applied mainly in the quality control of foodstuffs and in pharmaceutical industry. However, there are difficulties with purchase of these types of sensor. It seems that they are not stable enough for longstanding application.

Another potentiometric taste sensor consists of five lipid, lipid like-polymer membrane electrodes [7,9]. It is sensitive to bitter, sour and salt substances and not sensitive to sweet agents. This sensor was applied for quality control of different kinds of soft drinks [7].

A sensor called "electronic tongue" based on impulse voltammetry consists of four electrodes of different metals, a reference electrode and a counter electrode [4]. It was applied for recognition of different teas, milk, drinking water and different fruit juices [4,5]. This sensor requires expensive apparatus (potentiostat and generator) and complex numerical analysis (principal component analysis, PCA).

All these taste sensors have rather complicated construction and unsatisfactory stability and reproducibility. Therefore, the all-solid-state electrodes (ASSEs) are investigated in the present work for easy application as a base for taste sensor. Since ASSEs do not have liquid inner solution they seem to have simpler construction [12] than traditional ion selective electrodes (ISEs) [11-13].

An all-solid-state electrode (ASSE) consists of two layers covering glassy carbon disc. One is electroactive polymer, poly(3,4-ethylenedioxythiophene), doped with polystyrene sulfonate (PEDOT/PSS). The other one (outer) is made from polyvinyl chloride (PVC) containing lipophilic compound and plasticizer.

Since it was previously shown [7] that potential responses of ISEs with positively charged membranes are opposite to ISEs responses with neutral PVC membranes, the aim of this work is to test if this holds true also for ASSEs. Hence, four ASSEs containing two kinds of lipophilic compounds in PVC membranes: positively charged (benzyldimethyltetradecylammonium chloride, dodecyltrimethylammonium bromide) and neutral 
(palmitic acid, stearic acid) were tested in different solutions (hydrochloric acid, citric acid, acetic acid). ASSEs stability and sensitivity to sour substances were examined. The effect of thickness of lipophilic compound-PVC membrane and of electroactive polymer layer $\left(d_{\mathrm{PVC}}, d_{\mathrm{PEDOT}}\right.$, respectively) on electrode responses was examined. The influence of two different mass ratios of lipophilic compound to PVC on responses of ASSEs in sour substances solutions was discussed. The first mass ratio (ASSEs I), was proposed by Bobacka [12], the latter (ASSEs II) was chosen from a set of mass ratios investigated by us.

\section{Experimental}

\subsection{Materials}

Dodecyltrimethylammonium bromide, palmitic and stearic acids, dioctyl phenylphosphonate (DOPP), the monomer 3,4-ethylenedioxythiophene (EDOT) and poly(sodium 4-styrenesulfonate) (NaPSS, MM $\approx 70,000)$ were from Aldrich. Anhydrous benzyldimethyltetradecylammonium chloride and high molecular weight PVC were from Fluka. Tetrahydrofuran (THF) was distilled under $\mathrm{LiAlH}_{4}$. The other chemicals used were of analytical grade. Distilled water was used to prepare all solutions.

\subsection{Electrochemical synthesis of PEDOT}

Three-electrode electrochemical cell was used with a glassy carbon (GC) disc working electrode $\left(\right.$ area $\left.=0.12 \mathrm{~cm}^{2}\right)$, platinum mesh auxiliary electrode, and $\mathrm{Ag} / \mathrm{AgCl} / \mathrm{Cl}^{-}(0.1 \mathrm{M} \mathrm{KCl})$ reference electrode. Prior to polymerization GC working electrodes were polished with $0.05 \mu \mathrm{m}$ alumina, rinsed with distilled water, and cleaned ultrasonically. PEDOT was deposited on the GC working electrodes by galvanostatic electrochemical polymerization from a deoxygenated solution containing $0.01 \mathrm{M}$ EDOT and $0.1 \mathrm{M} \mathrm{NaPSS}$ as supporting electrolyte at constant electric potential $(850 \mathrm{mV} /$
$\mathrm{Ag} / \mathrm{AgCl} / 0.1 \mathrm{M} \mathrm{KCl}$ ). Polymerization charges of 10 and $20 \mathrm{mC}$ were produced, which refer to $0.5 \mu \mathrm{m}$ and $1.0 \mu \mathrm{m}$ thicknesses of PEDOT doped with polystyrenesulfonate layer, respectively.

\subsection{All-solid-state electrodes preparation}

ASSE were prepared [12] by coating GC/ PEDOT electrodes with an ion selective lipophilic compound-polymer membrane of the following compositions:

ASSE I: $2 \mathrm{mg}$ of appropriate lipophilic compound, $60 \mathrm{mg}$ PVC (mass ratio: lipophilic compound $/ \mathrm{PVC}=0.0330), 120 \mathrm{mg}$ DOPP in $1.5 \mathrm{~mL}$ THF.

ASSE II: $2.5 \mathrm{mg}$ of appropriate lipophilic compound, $300 \mathrm{mg}$ PVC (mass ratio: lipophilic compound $/ \mathrm{PVC}=0.0083), \quad 483.5 \mathrm{mg}$ DOPP in $10 \mathrm{~mL}$ THF.

The solution was put on the electrodes covered with PEDOT/PSS layer and dried. The obtained lipophilic compound-polymer membrane films of $10 \mu \mathrm{m}$ and $100 \mu \mathrm{m}$ thickness were transparent and colourless. All ASSE were conditioned in $0.1 \mathrm{M} \mathrm{KCl}$ for 1 day before use.

\subsection{Experimental setup}

The four ASSE and a reference electrode $\mathrm{Ag} / \mathrm{AgCl} / \mathrm{Cl}^{-}(3 \mathrm{M} \mathrm{KCl})$ were immersed in the appropriate sour solution $\left(10^{-4}-10^{-1} \mathrm{M}\right)$. The electrode responses were measured using highinput-impedance voltmeter Hach EC30 (23 \pm $2{ }^{\circ} \mathrm{C}$ ) (Fig. 1). The electrodes were conditioned in $0.1 \mathrm{M} \mathrm{KCl}$ prior to and between measurements.

\section{Results and discussion}

The experimental setup consists of four ASSEs, each with different lipophilic compound in PVC. Similarly as in case of ISEs [7] lipophilic compound-PVC membranes can be divided into two groups: 


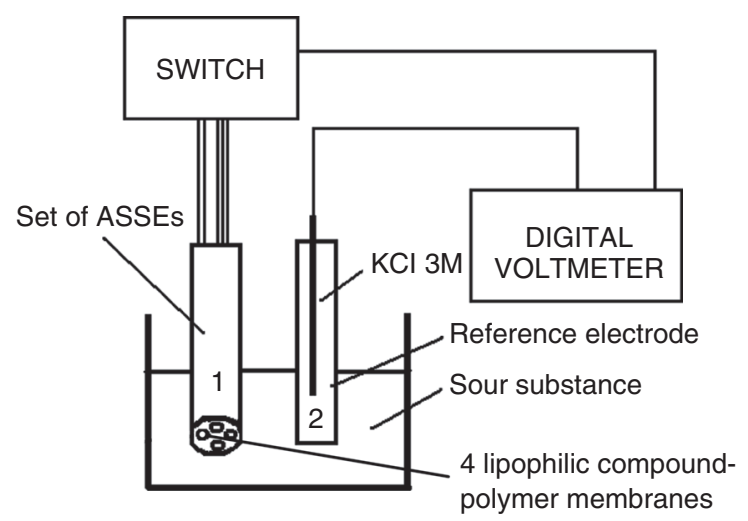

Fig. 1. Experimenal setup: 1 -four ASSEs, $2-\mathrm{Ag} / \mathrm{AgCl} /$ $\mathrm{Cl}^{-}$reference electrode.

1. positively charged membranes containing benzyldimethyltetradecylammonium chloride or dodecyltrimethylammonium bromide, and

2. neutral membranes with palmitic or stearic acid.
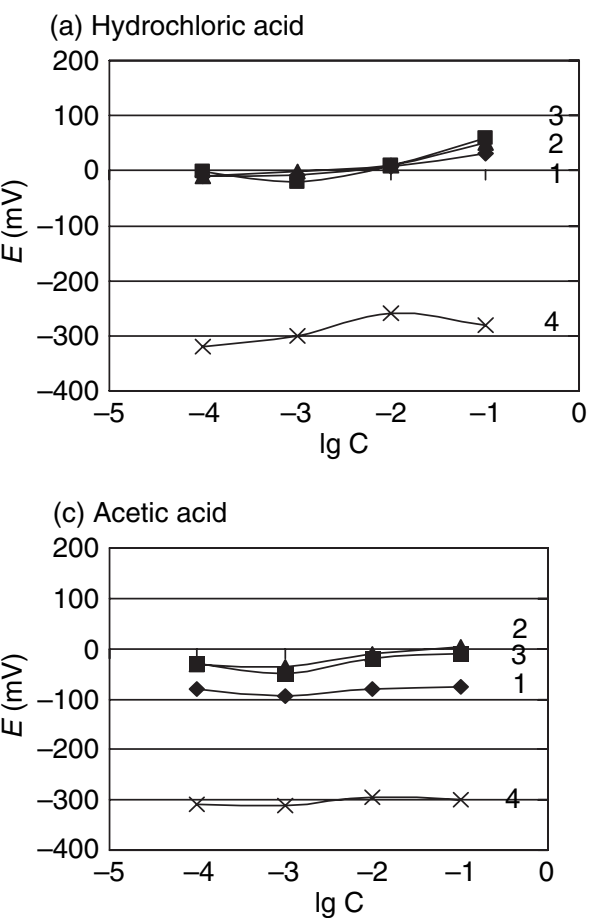

The membranes of group 2 can be considered as neutral since these lipohilic compounds are not dissociated in aqueous solution.

The results obtained with ASSEs of two lipophilic compound/PVC mass ratios are discussed (ASSE I and ASSE II, see "Experimental" section).

\subsection{Sensitivity of ASSE I}

Sensitivity of ASSE I to sour compound concentrations is presented in Fig. 2. In case of hydrochloric acid the $E=f(C)$ relationships for electrodes with positively charged membranes are linear with the slopes of 18.9 and 25.4 for benzyldimethyltetradecylammonium chloride (Fig. 2a, curve 1) and dodecyltrimethylammonium bromide (Fig. 2a, curve 2), respectively. The response of neutral membrane electrodes is

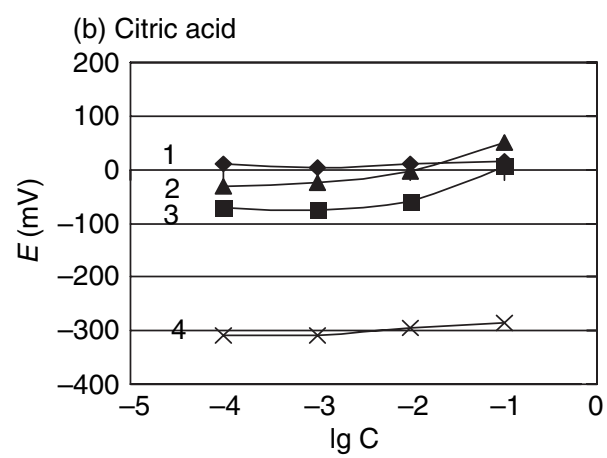


(a)

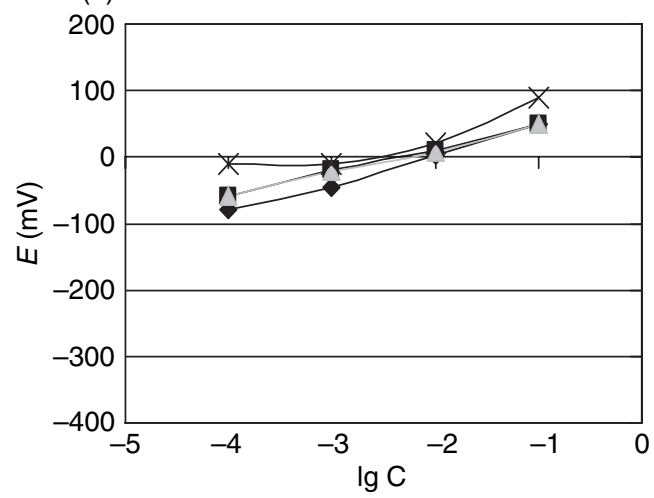

(b)

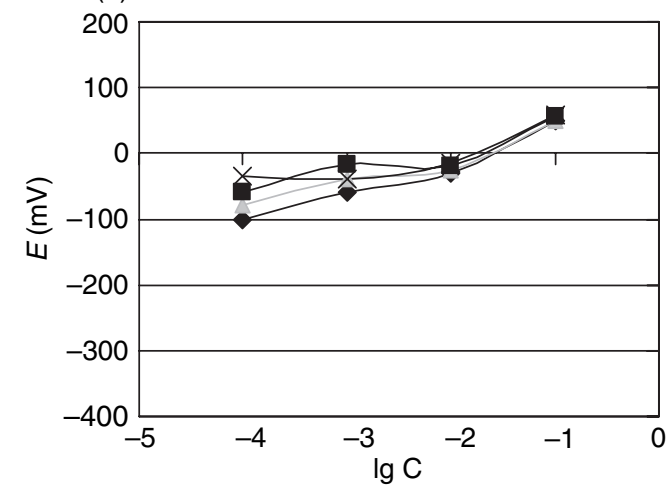

Fig. 3. Stability of ASSE I with stearic acid of different $d_{\mathrm{PEDOT}}$ and $d_{\mathrm{PVC}}$ as a function of citric acid concentration $(C)$ after: $\boldsymbol{\Delta}-1$ day, $\mathbf{\square}-2$ days, -3 days, $\mathbf{x}-8$ days; (a) $d_{\mathrm{PVC}}=10 \mu \mathrm{m}, d_{\mathrm{PEDOT}}=0.5 \mu \mathrm{m}$, (b) $d_{\mathrm{PVC}}=10 \mu \mathrm{m}, d_{\mathrm{PEDOT}}=$ $1.0 \mu \mathrm{m}$.

not linear (Fig. 2a, curves 3 and 4). ASSEs I containing dodecyltrimethyl ammonium bromide (curve 2 in Fig. 2b) and palmitic acid (curve 3 in Fig. 2b) in citric acid solution are only sensitive in the range of concentration of $10^{-1}-10^{-2} \mathrm{M}$. In acetic acid solutions, this sensitivity (curves 2 and 3 in Fig. 2c) is even lower. It should be noted that electrode responses of ASSE I with stearic acid (curve 4 in Fig. $2 \mathrm{a}-\mathrm{c}$ ) have much lower potential values than the other ASSEs I in all sour solutions examined.

These results suggest that ASSEs I are not sensitive enough to sour substances concentration, especially in case of citric and acetic acids solutions. Since those acids are mainly used in food industry it seems that ASSEs I are not suitable for sour taste sensing.

\subsection{Stability of ASSES I}

Stability of ASSEs I was also investigated (Figs. 3 and 4). The results obtained for ASSEs I

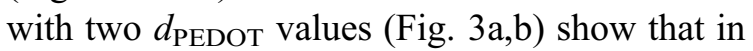
both cases the changes of $E=f(C)$ relationships with time are similar. This means that PEDOT/ PSS thickness has influence neither on stability nor on sensitivity of ASSEs I.
ASSE I with stearic acid of higher $d_{\mathrm{PVC}}$ and $d_{\text {PEDOT }}$ thicknesses was also tested in citric acid solution (Fig. 4). As it can be seen the stability of electrode was improved but unfortunately its sensitivity decreased. This confirms a general phenomenon well known in the field of membrane science: improving stability brings about loss of sensitivity [14].

To improve sensitivity of ASSEs, the different mass ratios of lipophilic compound/PVC were tested. ASSEs II were characterized by better sensitivity in comparison to the other ASSEs investigated.

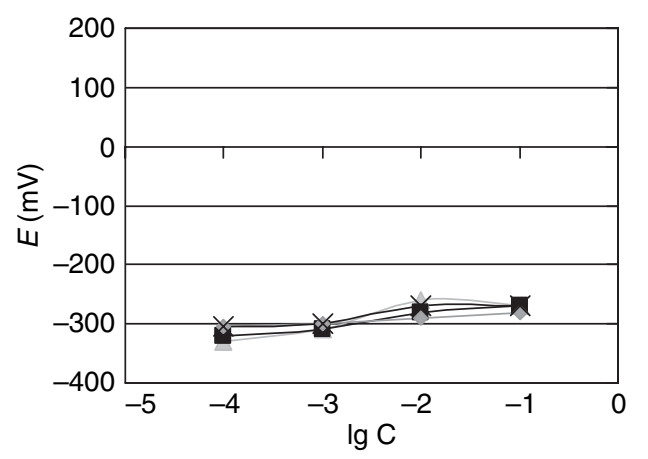

Fig. 4. Stability of ASSE I with stearic acid of $d_{\mathrm{PVC}}=$ $100 \mu \mathrm{m}, d_{\text {PEDOT }}=1.0 \mu \mathrm{m}$ as a function of citric acid concentration $(C)$ after: $\boldsymbol{\Delta}-1$ day, $\mathbf{\square}-2$ days, -3 days, $\mathbf{x}-8$ days. 

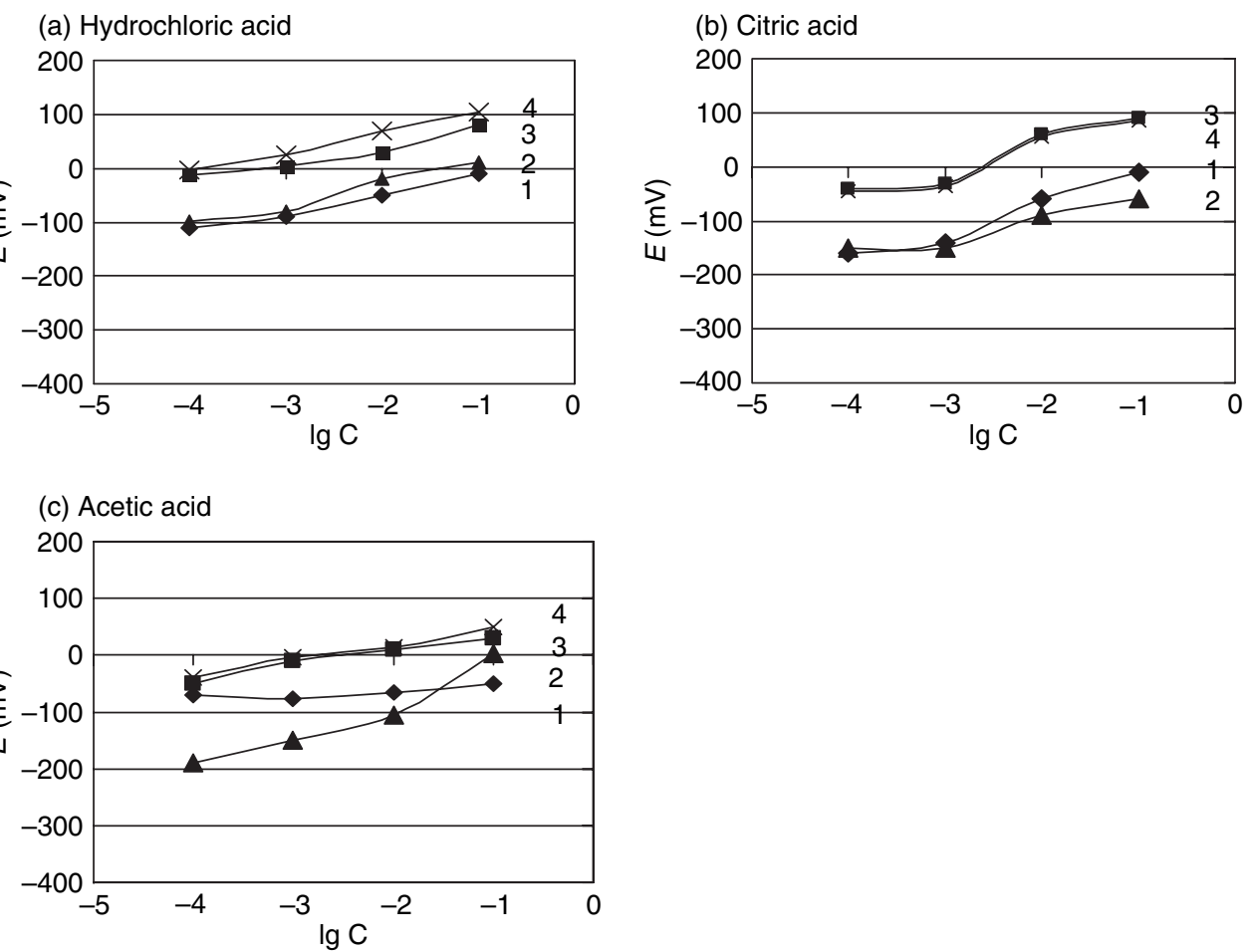

Fig. 5. Electric potential $(E)$ changes of ASSE II $\left(d_{\mathrm{PVC}}=10 \mu \mathrm{m}, d_{\mathrm{PEDOT}}=0.5 \mu \mathrm{m}\right)$ with $\bullet$ - benzyldimethyltetradecylammonium chloride (1), $\mathbf{\Delta}$ - dodecyltrimethyl ammonium bromide (2), $\mathbf{\square}$ - palmitic acid (3), $\mathbf{x}$ - stearic acid (4) as a function of sour substances concentration $(C)$.

\subsection{Sensitivity of ASSE II}

Sensitivity of ASSE II was tested in sour solutions of different concentrations (Fig. 5). In the case of hydrochloric acid solutions (Fig. 5a) straight lines of $E=f(C)$ are observed for each electrode used. The slopes of $E=f(C)$ are the following: 32.5, 39.3, 34.4, 41.6 for benzyldimethyltetradecylammonium chloride (1), dodecyltrimethylammonium bromide (2), palmitic acid (3) and stearic acid (4), respectively. They are much higher than slopes obtained for ASSEs I (Fig. 2a). This means that ASSEs II are more sensitive than ASSEs I. The other effect observed was that sensitivity of positively charged and neutral membranes to concentration of hydrochloric acid is rather similar.

The shapes of the $E=f(c)$ curves obtained in citric acid solutions (Fig. 5b) are different from those obtained in hydrochloric acid. The membrane electrodes are sensitive to citric acid concentration only in the range of $10^{-2}-10^{-3} \mathrm{M}$. In case of acetic acid solution (Fig. 5c), the $E=f(C)$ relation is linear for ASSE II with neutral membranes (slope 28.1 and 30.8 for palmitic acid (3)

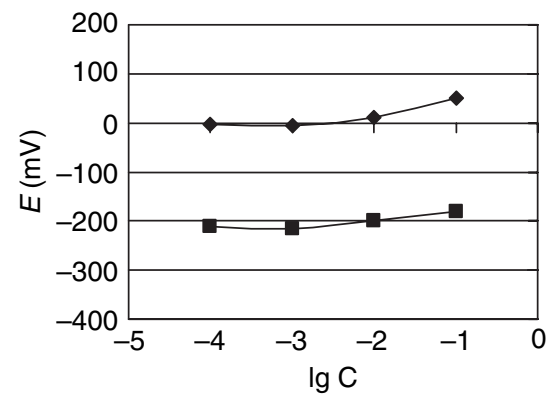

Fig. 6. Electric potential $(E)$ of ASSE II with stearic acid concentrations $(C)$ of $d_{\mathrm{PVC}}=100 \mathrm{~mm}$, $d_{\text {PEDOT }}=0.5 \mu \mathrm{m}$, or $(\square) d_{\text {PEDOT }}=1.0 \mu \mathrm{m}$. 
(a)

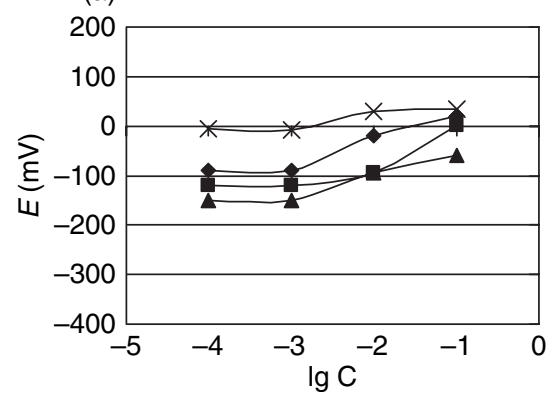

(b)

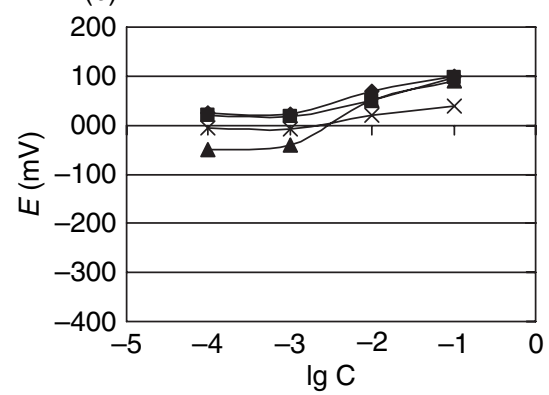

Fig. 7. Electric potential (E) of ASSE II with (a) dodecyltrimethylammonium bromide, $d_{\mathrm{PVC}}=10 \mathrm{~mm}, d_{\mathrm{PEDOT}}=0.5 \mathrm{~mm}$ in citric acid solutions, (b) stearic acid, $d_{\mathrm{PVC}}=10 \mathrm{~mm}, d_{\mathrm{PEDOT}}=0.5 \mathrm{~mm}$ in citric acid solution of different concentrations $(C)$ after: $\mathbf{\Lambda}-1$ day, $\mathbf{\square}-2$ days, -3 days, $\mathbf{x}-8$ days.

and stearic acid (4), respectively). Responses of ASSE II with positively charged membranes (curves 1 and 2 in Fig. 5c) are not linear.

Straight line relationship of $E=f(C)$ for $\mathrm{HCl}$ and its higher sensitivity in comparison to citric and acetic acids suggest that ASSEs responses are due to $\mathrm{H}^{+}$concentration directly. $\mathrm{HCl}$ molecules are fully dissociated in water $\left(\mathrm{p} K_{\mathrm{a}}=-7\right)$, meanwhile this is not the case of citric and acetic acids. $\mathrm{p} K_{\mathrm{a}}$ values for citric acid are the following: $\mathrm{p} K_{\mathrm{a} 1}=3.13, \mathrm{p} K_{\mathrm{a} 2}=4.76, \mathrm{p} K_{\mathrm{a} 3}=6.40$ and for acetic acid $\mathrm{p} K_{\mathrm{a}}$ is equal to 4.76 [15].

All these results indicate that such electrodes might be used for $\mathrm{pH}$ determination after calibration before measurements.

The influence of $d_{\text {PEDOT }}$ on ASSE II sensitivity was also examined. Two different $d_{\text {PEDOT }}$ values were used: $0.5 \mu \mathrm{m}$ and $1.0 \mu \mathrm{m}$ (Fig. 6). As it can be seen the shape of $E=f(C)$ function is similar for both $d_{\text {PEDOT }}$ values. These results confirm that PEDOT/PSS thickness has no influence on ASSE II sensitivity in citric acid solutions, as in the case of ASSE I. The shift of responses results from the inner potential of the electroactive polymer.

\subsection{Stability of ASSE II}

Stability of ASSE II membrane electrode with dodecyltrimethylammonium bromide (group 1) and with stearic acid (group 2) was also tested. The potential responses of positively charged (Fig. 7a) and neutral membrane electrodes (Fig. 7b) are changing with time. Systematic shift of electrode responses is observed for each case. The reason of this shift is not clear; however, it is very likely that inner membrane PEDOT, which is a cation-selective film, interferes with the response of the outer layer. This hypothesis seems to be confirmed by the examination of ASSEs I with thicker PVC outer layer.

\section{Conclusions}

Multichannel taste sensors with positively charged and neutral ion selective membranes (ISEs) were successfully applied in quality control of foodstuffs. Since these electrodes have liquid inner solutions, all-solid-state electrodes with positively charged membranes (benzyldimethyltetradecylammonium chloride, dodecyltrimethylammonium bromide) and neutral membranes (palmitic acid, stearic acid) were tested in selected sour solutions (hydrochloric acid, citric acid, acetic acid).

It was found that ASSEs with positively charged and neutral PVC membranes behave similarly in full contradiction to ISEs $[7,9]$. 
Straight line relationship of $E=f(C)$ for $\mathrm{HCl}$ and its higher sensitivity in comparison to citric and acetic acids suggest that ASSEs responses are due to $\mathrm{H}^{+}$concentration directly since $\mathrm{HCl}$ molecules are fully dissociated in water. This indicates that such electrodes can be used for $\mathrm{pH}$ determination provided that calibration is done before measurements.

ASSEs II of mass ratio of lipophilic compound/PVC proposed by us are more sensitive to tested sour substances than ASSEs I [12], but they are less stable.

The thickness of PEDOT has no influence on stability and sensitivity of tested systems. On the contrary, the higher thickness of lipophilic compound - PVC membrane improves electrodes stability but decreases their sensitivity to sour substances.

At the present stage it is difficult to judge if ASSEs can be used for taste sensing. The stability and sensitivity should be improved. It is planned to test ASSEs with PEDOT doped with anion selective additive, e.g. perchlorate ion $\left(\mathrm{PEDOT} / \mathrm{ClO}_{4}^{-}\right)$which should remove time-dependent shifting effects of electrode responses.

\section{References}

[1] B. Lindemann, Physiol. Rev., 76 (1996) 719-765.

[2] PN-ISO 3972, Sens. Anal. Methodol., 1998.

[3] K. Toko. Mater. Sci. Eng., C4 (1996) 69-92.

[4] F. Winquist, C. Krantz-Rülcker, P. Wide and J. Lundström, Meas. Sci. Technol., 9 (1998) 1937-1946.

[5] F. Winquist, P. Wide and I. Lundström. Anal. Chim. Acta, 357 (1997) 21-31.

[6] K. Toko, T. Matsuno, K. Yamafuji, K. Hayashi, H. Ikezaki, K. Sato, R. Toukubo and S. Kawarai, Biosens. Bioelectron., 9 (1994) 359-364.

[7] M. Szpakowska, A. Magnuszewska and J. Szwacki, J. Membr. Sci., 273 (2006) 116-123.

[8] K. Toko, Biosens. Bioelectron., 13 (1998) 701-709.

[9] J. Szwacki, A. Lisowska-Oleksiak and M. Szpakowska, Desalination, 198 (2004) 1-7.

[10] P. Ciosek and W. Wróblewski, Analyst, 132 (2007) 963-978.

[11] J. Bobacka, A. Lewenstam and A. Ivaska, Electroanal. Chem., 509 (2001) 27-30.

[12] J. Bobacka, Anal. Chem., 71 (1999) 4932-4937.

[13] L. Lvova, S. Shin Kim, A. Legin, Y. Vlasov, J. Soo Yang, G. Sig Cha and H. Nam, Anal. Chim. Acta, 468 (2002) 303-314.

[14] M. Mulder, Basic principles of membrane technology, Kluwer, London, 1991.

[15] R.C. Weast, M.J. Astle (Eds.), Handbook of Chemistry and Physics, CRC Press, Boca Raton, FL, 2000. 